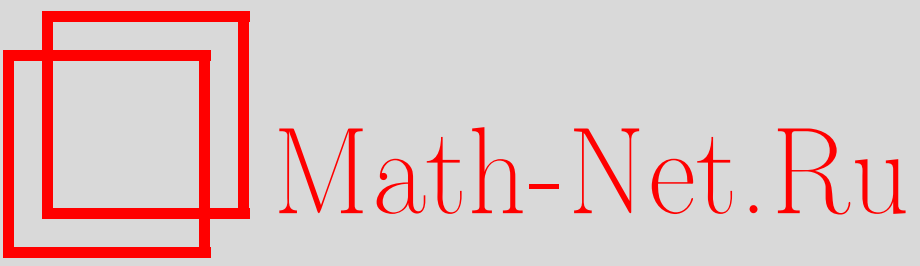

Р. Каллош, Демократическое описание $D=10$ суперсимметрии, $T M \Phi, 2001$, том 128, номер 3, 422-438

DOI: https://doi.org/10.4213/tmf506

Использование Общероссийского математического портала Math-Net.Ru подразумевает, что вы прочитали и согласны с пользовательским соглашением

http://www.mathnet.ru/rus/agreement

Параметры загрузки:

IP : 54.224 .187 .69

26 апреля 2023 г., 05:50:15 


\author{
ТЕОРЕТИЧЕСКАЯ \\ И МАТЕМАТИЧЕСКАЯ \\ ФИЗИКА \\ Том 128, № 3 \\ сентябрь, 2001
}

(C) 2001 г.

Р. Каллош*

\title{
ДЕМОКРАТИЧЕСКОЕ ОПИСАНИЕ $D=10$ СУПЕРСИММЕТРИИ
}

Исследуется обобщение IIA/В супергравитации, в котором теория зависит от всех рамон-рамоновских $(\mathrm{R}-\mathrm{R})$ потенциалов $C^{(p)}, p=0,1, \ldots, 9$, и представляет собой эфофективное полевое действие суперструнной теории типа IIA/B. В случае суперструны IIA равноправие $\mathrm{R}-\mathrm{R}$-потенциалов явно нарушается наложением условия $p \leqslant 3$ либо $p \geqslant 5$, что позволяет написать новое объемное действие, которое может быть присоединено к $N=1$ суперсимметричным бранным действиям.

Посвяшается 75-летию Владимира Яковлевича Файнберга.

\section{1. ВВЕДЕНИЕ}

Эта статья основана на совместной работе с Эриком Бергсхоффом, Томасом Ортином, Дидериком Ростом и Антуаном Ван Пройеном [1]. Исходной мотивацией этой работы было построение суперсимметричных доменных стенок в $D=10$ струнной теории, которое могло бы пролить свет на струнное происхождение сценариев возникновения бранного мира. Однако в процессе исследования мы поняли, что все имеющиеся в литературе описания эффективной полевой теории для струн типа IIA/В оказались малоподходяшими для нашей цели. Это привело к идее ввести новые версии эффективных супергравитаций, отвечающих струнным теориям типа IIA/B.

Стандартная IIA безмассовая супергравитация включает рамон-рамоновские (R-R) потенциалы $C^{(1)}$ и $C^{(3)}$ и соответствуюшие калибровочно-инвариантные R-R-формы $G^{(2)}$ и $G^{(4)}$. Супергравитация типа IIB включает R-R-потенциалы $C^{(0)}, C^{(2)}$ и $C^{(4)}$ и соответствующие калибровочно-инвариантные R-R-формы $G^{(1)}, G^{(3)}$ и (самодуальную) $G^{(5)}$. С другой стороны, струнная теория допускает существование всех возможных $\mathrm{D} p$-бран, как четных, так и нечетных, включая даже такие экзотические браны, как 8-браны в теории IIA и 7-браны в теории IIB. Эти последние браны коразмерностей 1 и 2 являются специальными объектами, отличающимися во многих отношениях от остальных протяженных БПС-симметричных объектов, таких как $p$-браны с $0 \leqslant p \leqslant 6$, которые имеют коразмерность больше или равную трем. Основное отличие заключено в поведении полей форм на большом расстоянии $G^{(p+2)} \sim r^{p-8}$. Например, R-R-форма $G^{(10)}$ для 8-браны не убывает на бесконечности, а принимает на ней постоянное значение. Общепринято, что такие протяженные объекты не могут сушествовать сами по се-

* Department of Physics, Stanford University, Stanford, California, USA 
бе; их сушествование возможно только в присутствии ориентифолдных плоскостей [2]. Тем не менее сама возможность построения такой полной системы бран и форм в супергравитации не очевидна.

Некоторое время тому назад [3] стало понятно, что массивная IIA супергравитация, открытая Романсом [4], лежит в основе понимания пространственно-временной структуры 8-бран, являющихся доменными стенками в размерности $D=10$. Значительный прогресс в понимании 8-бранных решений был достигнут в работах [5], [6], в которых было найдено решение супергравитации в объеме. В работе [6] также обсуждалось введенное в работах [7], [8] описание космологической постоянной в терминах потенциала 9-формы. В работе [9] было показано, что стандартное 8-бранное действие, присоединенное к потенциалу 9-формы, приводит ко второму сценарию Рэндалла-Сандрума [10] . Решения системы с присоединенными действиями в объеме и на бране, как было показано, автоматически удовлетворяют условиям скачка, а потому оказываются согласованными, по крайней мере с этой точки зрения. Наиболее значительной нерешенной проблемой оставалось построение явно суперсиммметричного описания присоединенной системы в объеме и на бране, как было сделано в работе [11]. В рамках такого описания могут быть найдены некоторые важные свойства доменных стенок, такие как расстояние между плоскостями, статус ненарушенной суперсимметрии в объеме и на бране и т.д. Можно также ожидать, что реализация такой конструкции бран, присоединенных к объему, приведет к лучшему пониманию фундаментальной природы протяженных объектов струнной теории.

Наш подход основан на обобшении предложенной в работе [11] пятимерной конструкции суперсимметричного действия в объеме с присоединенной браной. Конструкция работы [11] позволяет найти суперсимметричную реализацию возникновения бранного мира по сценарию Рэндалла и Сандрума [10]. Здесь мы повторяем построение работы [11] для размерности $D=10$ с целью лучшего понимания роли бран и плоскостей в струнной теории.

Чтобы разрешить противоречие между весьма ограниченным возможным полевым содержанием действия в объеме, где допустимы лишь R-R-формы нескольких низших порядков, и широким спектром бранных действий, которые могут содержать все возможные R-R-формы, будет предложена новая формулировка супергравитации IIA/B, вычисленная вплоть до четвертого порядка по фермионам. В частности, эта новая формулировка позволяет легко контролировать экзотические $G^{(0)}$ и $G^{(10)} \mathrm{R}$-R-формы, связанные с массой и космологической постоянной ${ }^{1)} D=10$ супергравитации. Это, в свою очередь, допускает явное исследование D8-O8 системы, описьвающей пару суперсимметричных доменных стенок, которые суть фундаментальные объекты струнной теории типа I'. Простым следствием теории является квантование параметра массы и космологической постоянной в струнных единицах. Можно ожидать, что новые эффективные теории $D=10$ суперсимметрии будут иметь и другие общие приложения, помимо описания суперсимметричных доменных стенок, которое послужило мотивацией для их

\footnotetext{
1) В данной работе космологическая постоянная есть квадрат массового параметра супергравитации типа IIA. Строго говоря, эта величина не является подлинной космологической постоянной, потому что она зависит от дилатонного множителя в системе Эйнштейна.
} 
введения.

Структура работы следуюшая. В разделе 2 рассматривается новая формулировка $D=10$ суперсимметрии в объеме. В п. 2.1 дается новое "демократическое" описание, основанное на псевдодействии, введенном в работе [12]. Это описание - одно из тех, в котором все R-R-потенциалы считаются равноправными, что приводит к тем же уравнениям движения, которые впервые появились в теории с присоединенными D-бранами [13]. В суперсимметричном случае такое полевое содержание и уравнения движения могут быть получены из суперпространственной формулировки теории [14], [15]. Тем не менее удается построить лиш псевдодействие, уравнения движения которого должны быть дополнены условиями дуальности. Это псевдодействие допускает унифицированное описание в терминах струн IIA/В и не содержит членов Черна-Саймонса. Условия дуальности не следуют из действия и накладываются дополнительно. Эти условия обобщают условие самодуальности, накладываемое на компоненты полевой 5-формы $G^{(5)}$ в теории типа IIB, и вводят набор соотношений между всеми полевыми формами, взаимодуальными по Ходжу. В теории типа IIB такая самодуальность не позволяет построить настоящее действие. Это препятствие сохраняется и в настоящей формулировке теории с псевдодействием и обобшенными условиями дуальности как для теории типа IIA, так и для теории типа IIB. В случае теории типа IIA условия дуальности связывают всегда различные полевые формы, и R-R-равноправие этих форм тем самым может быть нарушено сравнительно легко. Случай теории типа IIB оказывается более сложным, поскольку подобная конструкция невозможна ввиду самодуальности формы $G^{(5)}$. Правильное действие при этом может быть построено в нековариантной формулировке [16] или же с использованием конструкции вспомогательных полей Пасти, Сорокина и Тонина [17].

В п. 2.2 рассматривается нарушение самодуальности для струны типа IIA и строится суперсимметричное собственное действие, содержащее потенциалы $C^{(p)}, p=5,7,9$. Соотношения дуальности, возникающие из исходной формулировки, также не позволяют построить обобщение механизма из работы [11], в котором массовый параметр $G^{(0)}$ заменяется полем $G^{(0)}(x)$. Действительно, для этой процедуры необходимо сушествование независимого вспомогательного поля 9-формы. В демократическом подходе напряженность поля $G^{(10)}$ этой 9-формы связана непосредственно с $G^{(0)}$. Нарушение самодуальности, обсуждаемое в п. 2.2 , одновременно позволяет построить действие и допускает введение изменяющейся величины $G^{(0)}(x)$. Также оказывается, что в демократическом подходе не сохраняется нужная $\mathbb{Z}_{2}$-симметрия, играющая важную роль в механизме, предложенном в работе [11], в случае, когда массовый параметр отличен от нуля. Поэтому действие 8-браны, присоединенной к объему, строится именно в подходе, обсуждаемом в п. 2.2. Первая версия такой формулировки была предложена в работе [6]. В п. 2.3 получена струнная версия стандартной массивной IIA супергравитации Романса, в которой самодуальность также нарушена, но сохранены потенциалы $C^{(p)}$, $p=1,3$.

В разделе 3 обсуждается суперсимметрия на бране. Механизм суперсимметрии при этом один и тот же для всех бран, но добавляется действие лиш одной браны, и равноправие тем самым нарушается. Наконец, результаты обсуждаются в разделе 4 . В статье используются обозначения работы [1]. 


\section{2. СУПЕРСИММЕТРИЯ В ОБЪЕМЕ}

В стандартной формулировке $D=10$ IIA (безмассовой [18], [19], [20] и массивной [4]) и IIB $[21],[22]$ супергравитации включаются следуюшие поля:

$$
\begin{array}{ll}
\text { IIA : } & \left\{g_{\mu \nu}, B_{\mu \nu}, \phi, C_{\mu}^{(1)}, C_{\mu \nu \rho}^{(3)}, \psi_{\mu}, \lambda\right\}, \\
\text { IIB : } & \left\{g_{\mu \nu}, B_{\mu \nu}, \phi, C^{(0)}, C_{\mu \nu}^{(2)}, C_{\mu \nu \rho \sigma}^{(4)}, \psi_{\mu}, \lambda\right\} .
\end{array}
$$

В случае теории IIA массивная теория содержит дополнительньй массовый параметр $G^{(0)}=m$. В случае теории IIВ на напряженности поля 4-формы накладьвается дополнительное условие самодуальности. Оказывается, что $N=2$ суперсимметрия также может быть реализована на калибровочных R-R-полях старших порядков. Обычно такие поля вводятся с помошью соотношений дуальности. В случае, когда все R-R-потенциалы считаются равноправными, мы предлагаем в п. 2.1 новый подход, основанный на псевдодействии. Эта демократическая формулировка теории наиболее просто описывает динамику супергравитации в объеме, но оказьвается не слишком хорошо приспособленной для описания интересуюших нас явлений. Поэтому в п. 2.2 дается подправленная формулировка теории для случая IIА, где постоянный массовый параметр заменяется переменным полем. Соотношение меж ду этой формулировкой и стандартной IIA супергравитацией (с набором полей (2.1)) обсуждается в п. 2.3 .

2.1. Демократическое описание. Для того чтобы считать все R-R-потенциалы равноправными, мы предлагаем псевдодействие, содержашее всепотенциалы. При этом, разумеется, увеличивается число степеней свободы. Поскольку потенциалы $p$ - и $(8-$ $p$ )-форм обладают одинаковым числом степеней свободы, введение дуальных потенциалов удваивает число степеней свободы в R-R-секторе. Введение потенциала старшей степени $C^{(9)}$ в секторе IIA не меняет это утверждение, поскольку этот потенциал (9-форма) вообще не имеет непрерывных степеней свободы и должен рассматриваться как потенциал, дуальный постоянному параметру массы $G^{(0)}=m$. Удвоение числа степеней свободы устраняется наложением условий, связываюших потенциалы низших и высших порядков. Эта новая суперсимметричная формулировка, подсказанная бозонной конструкцией, предложенной в работе [23], связана в случае IIB супергравитации также и с конструкцией псевдодействия, предложенной в работе [12].

В работе [12] с помошью псевдодействия выводились мнемонические правила вывода уравнений движения. Приставка "псевдо" означает, что не все уравнения движения следуют из вариаций полей в псевдодействии. Полный набор уравнений движения можно получить, только пополнив набор уравнений движения, вытекаюших из псевдодействия, дополнительной связью, накладываемой принудительно и не следующей из псевдодействия. Конструкция псевдодействия в данной работе обобщает результаты работ [23], [12], поскольку, во-первых, случаи IIA и IIB объединяются в конструкции в единый объект, все R-R-потенциалы включены в псевдодействие и, во-вторых, дается описание массивной IIA теории с помошью включения потенциала $C^{(9)}$ 9-формы и дуального к нему постоянного массового параметра $G^{(0)}=m$.

Псевдодействие включает в себя расширенный состав полей:

$$
\begin{array}{ll}
\text { IIA : } & \left\{g_{\mu \nu}, B_{\mu \nu}, \phi, C_{\mu}^{(1)}, C_{\mu \nu \rho}^{(3)}, C_{\mu \ldots \rho}^{(5)}, C_{\mu \ldots \rho}^{(7)}, C_{\mu \ldots \rho}^{(9)}, \psi_{\mu}, \lambda\right\}, \\
\text { IIB : } & \left\{g_{\mu \nu}, B_{\mu \nu}, \phi, C^{(0)}, C_{\mu \nu}^{(2)}, C_{\mu \ldots \rho}^{(4)}, C_{\mu \ldots \rho}^{(6)}, C_{\mu \ldots \rho}^{(8)}, \psi_{\mu}, \lambda\right\} .
\end{array}
$$


Принимается, что в случае IIA включаются фермионы обеих киральностей, в то время как в случае IIB фермионы удовлетворяют условиям

$$
\Gamma_{11} \psi_{\mu}=\psi_{\mu}, \quad \Gamma_{11} \lambda=-\lambda
$$

При наложении этих условий фермионы становятся дублетами, соответствуюший индекс будет опускаться. Явная формула для псевдодействия имеет вид ${ }^{2}$ )

$$
\begin{aligned}
S_{\text {pseudo }}= & -\frac{1}{2 \kappa_{10}^{2}} \int d^{10} x \sqrt{-g}\left\{e ^ { - 2 \phi } \left[R(\omega(e))-4(\partial \phi)^{2}+\frac{1}{2} H \cdot H-\right.\right. \\
& \left.-2 \partial^{\mu} \phi \chi_{\mu}^{(1)}+H \cdot \chi^{(3)}+2 \bar{\psi}_{\mu} \Gamma^{\mu \nu \rho} \nabla_{\nu} \psi_{\rho}-2 \bar{\lambda} \Gamma^{\mu} \nabla_{\mu} \lambda+4 \bar{\lambda} \Gamma^{\mu \nu} \nabla_{\mu} \psi_{\nu}\right]+ \\
& \left.+\sum_{n=0,1 / 2}^{5,9 / 2} \frac{1}{4} G^{(2 n)} \cdot G^{(2 n)}+\frac{1}{2} G^{(2 n)} \cdot \Psi^{(2 n)}\right\}+ \\
& + \text { члены четвертого порядка по фермионам. }
\end{aligned}
$$

Суммирование в псевдодействии идет по целым числам $n=0,1, \ldots, 5$ в случае IIА и по полуцелым $n=1 / 2,3 / 2, \ldots, 9 / 2$ в случае IIВ. Под знаком суммы первым указано низшее значение переменной суммирования для полей IIA, а затем для полей IIB. Более того,

$$
\frac{1}{2 \kappa_{10}^{2}}=\frac{g^{2}}{2 \kappa^{2}}=\frac{2 \pi}{\left(2 \pi \ell_{s}\right)^{8}}
$$

где $\kappa^{2}$ - константа связи для присоединенной физической гравитации, $g$ - струнная константа связи, а $\ell_{s}=\sqrt{\alpha^{\prime}}-$ длина струны. Для удобства обозначений все потенциалы и напряженности полей объединяются в формальные суммы

$$
\mathbf{G}=\sum_{n=0,1 / 2}^{5,9 / 2} G^{(2 n)}, \quad \mathbf{C}=\sum_{n=1,1 / 2}^{5,9 / 2} C^{(2 n-1)} .
$$

Напряженности бозонных полей в $\mathbf{C}$-базисе записываются в виде

$$
H=d B, \quad \mathbf{G}=d \mathbf{C}-d B \wedge \mathbf{C}+G^{(0)} \mathbf{e}^{B},
$$

где считается, что каждое уравнение содержит лишш один член из формальных сумм (2.4) (т.е. извлекаются только лишш нужные комбинации полей). Тогда соответствующие тождества Бианки имеют вид

$$
d H=0, \quad d \mathbf{G}-H \wedge \mathbf{G}=0 .
$$

Сушествует и альтернативный базис для R-R-потенциалов, который можно назвать A-базисом, который оказывается полезным в некоторых случаях. Этот базис связан с только что введенным $\mathbf{C}$-базисом соотношениями

$$
\mathbf{A}=\mathbf{C} \wedge \mathbf{e}^{-B}
$$

\footnotetext{
2) Здесь частично используются обозначения в терминах форм из работы [1].
} 
с калибровочными преобразованиями

$$
\delta \mathbf{A}=d \mathbf{\Lambda}-G^{(0)} \Lambda^{\mathrm{NS}}-\mathbf{A} \wedge d \Lambda
$$

В этом базисе напряженности R-R-полей имеют вид

$$
\mathbf{G}=\left(d \mathbf{A}+G^{(0)}\right) \wedge \mathbf{e}^{B}
$$

Два главных преимушества этого базиса заключаются в том, что в нем R-R-потенциалы участвуют в напряженностях полей только через свои производные и стандартный член Весса-Зумино действия D $p$-браны не содержит NS-NS 2-формы $B$. В действии типа IIB, записанном в этом базисе, явно видна инвариантность относительно постоянных сдвигов скалярного R-R-поля (аксиона). Существование такого базиса обеспечивает возможность обобщенной размерной редукции по Шерку и Шварцу [6].

В настояшем пункте мы считаем, что $G^{(0)}=m$ есть постоянный массовый параметр IIA супергравитации. В случае теории IIB во всех уравнениях следует положить постоянное поле $G^{(0)}$ равным нулю. Спиновая связность в ковариантной производной $\nabla_{\mu}$ задается своей десятиадной (zehnbein) составляющей: $\omega_{\mu}{ }^{a b}=\omega_{\mu}{ }^{a b}(e)$. Члены взаимодействия бозонных полей с фермионами задаются билинейными комбинациями $\chi^{(1,3)}$ и $\Psi^{(2 n)}$ :

$$
\begin{aligned}
\chi_{\mu}^{(1)}= & -2 \bar{\psi}_{\nu} \Gamma^{\nu} \psi_{\mu}-2 \bar{\lambda} \Gamma^{\nu} \Gamma_{\mu} \psi_{\nu}, \\
\chi_{\mu \nu \rho}^{(3)}= & \frac{1}{2} \bar{\psi}_{\alpha} \Gamma^{[\alpha} \Gamma_{\mu \nu \rho} \Gamma^{\beta]} \mathcal{P} \psi_{\beta}+\bar{\lambda} \Gamma_{\mu \nu \rho}{ }^{\beta} \mathcal{P} \psi_{\beta}-\frac{1}{2} \bar{\lambda} \mathcal{P} \Gamma_{\mu \nu \rho} \lambda, \\
\Psi_{\mu_{1} \ldots \mu_{2 n}}^{(2 n)}= & \frac{1}{2} e^{-\phi} \bar{\psi}_{\alpha} \Gamma^{[\alpha} \Gamma_{\mu_{1} \ldots \mu_{2 n}} \Gamma^{\beta]} \mathcal{P}_{n} \psi_{\beta}+\frac{1}{2} e^{-\phi} \bar{\lambda} \Gamma_{\mu_{1} \ldots \mu_{2 n}} \Gamma^{\beta} \mathcal{P}_{n} \psi_{\beta}- \\
& -\frac{1}{4} e^{-\phi} \bar{\lambda} \Gamma_{\left[\mu_{1} \ldots \mu_{2 n-1}\right.} \mathcal{P}_{n} \Gamma_{\left.\mu_{2 n}\right]} \lambda .
\end{aligned}
$$

Здесь использованы определения

$$
\begin{array}{lll}
\text { IIA : } & \mathcal{P}=\Gamma_{11}, & \mathcal{P}_{n}=\left(\Gamma_{11}\right)^{n} ; \\
\text { IIB : } & \mathcal{P}=-\sigma^{3}, & \mathcal{P}_{n}=\left\{\begin{array}{lll}
\sigma^{1}, & n+1 / 2 \\
i \sigma^{2} & n+1 / 2 & \text { четно, }
\end{array}\right.
\end{array}
$$

При этом фермионы должны удовлетворять условию

$$
\Psi^{(2 n)}=(-1)^{\operatorname{Int}[n]+1} \star \Psi^{(10-2 n)},
$$

следуюшему из тождества для Г-матриц.

Поскольку включены все R-R-потенциалы, число степеней свободы в R-R-секторе удваивается. Каждый R-R-потенциал приводит к соответствуюшему уравнению движения:

$$
d \star\left(G^{(2 n)}+\Psi^{(2 n)}\right)+H \wedge \star\left(G^{(2 n+2)}+\Psi^{(2 n+2)}\right)=0 .
$$


Различные потенциалы должны быть связаны между собой, чтобы полное число степеней свободы осталось прежним. Для этого в уравнениях движения, порождаемых псевдодействием (2.3), наложим дополнительные условия дуальности:

$$
G^{(2 n)}+\Psi^{(2 n)}=(-1)^{\operatorname{Int}[n]} \star G^{(10-2 n)} .
$$

Ввиду этих условий действие (2.3) не является полным. Вместо этого его следует рассматривать как мнемоническое правило для получения уравнений движения теории. Как обычно, соотношение дуальности связывает между собой тождества Бианки и уравнения движения для дуальных потенциалов. Поэтому демократическая формулировка может быть интерпретирована как самодуальная, поскольку (2.7) налагает ограничения на поля, указанные в списке (2.2).

Во-первых, псевдодействие (2.3) становится суперсимметрично-инвариантным, если наложить условия дуальности (2.7) после вариации действия. Правила суперсимметрии имеют вид (с точностью до членов третьего порядка по фермионам)

$$
\begin{aligned}
\delta_{\epsilon} e_{\mu}{ }^{a}= & \bar{\epsilon} \Gamma^{a} \psi_{\mu}, \\
\delta_{\epsilon} \psi_{\mu}= & \left(\partial_{\mu}+\frac{1}{4} \psi_{\mu}+\frac{1}{8} \mathcal{P} H_{\mu}\right) \epsilon+\frac{1}{16} e^{\phi} \sum_{n=0,1 / 2}^{5,9 / 2} \frac{1}{(2 n) !} G^{(2 n)} \Gamma_{\mu} \mathcal{P}_{n} \epsilon, \\
\delta_{\epsilon} B_{\mu \nu}= & -2 \bar{\epsilon} \Gamma_{[\mu} \mathcal{P} \psi_{\nu]}, \\
\delta_{\epsilon} C_{\mu_{1} \ldots \mu_{2 n-1}}^{(2 n-1)}= & -e^{-\phi} \bar{\epsilon} \Gamma_{\left[\mu_{1} \ldots \mu_{2 n-2}\right.} \mathcal{P}_{n}\left((2 n-1) \psi_{\left.\mu_{2 n-1}\right]}-\frac{1}{2} \Gamma_{\left.\mu_{2 n-1}\right]} \lambda\right)+ \\
& +(n-1)(2 n-1) C_{\left[\mu_{1} \ldots \mu_{2 n-3}\right.}^{(2 n-3)} \delta_{\epsilon} B_{\left.\mu_{2 n-2} \mu_{2 n-1}\right]}, \\
\delta_{\epsilon} \lambda= & \left(\not \partial \phi+\frac{1}{12} \not H \mathcal{P}\right) \epsilon+\frac{1}{8} e^{\phi} \sum_{n=0,1 / 2}^{5,9 / 2}(-1)^{2 n} \frac{5-2 n}{(2 n) !} G^{(2 n)} \mathcal{P}_{n} \epsilon, \\
\delta_{\epsilon} \phi= & \frac{1}{2} \bar{\epsilon} \lambda,
\end{aligned}
$$

где $\epsilon$ - спинор, сходный с $\psi_{\mu}$, т.е. $\Gamma_{11} \epsilon=\epsilon$ в теории IIВ. Заметим, что для полуцелых $n$ (случай IIB) вышеприведенные правила суперсимметрии в точности воспроизводят правила, указанные в уравнении (1.1) работы [24].

Во-вторых, псевдодействие (2.3) также инвариантно относительно обычных бозонных NS-NS и R-R калибровочных преобразований с соответствуюшими параметрами $\Lambda^{\mathrm{NS}}$ и $\Lambda^{(2 n)}$ :

$$
\delta_{\Lambda} B=d \Lambda^{\mathrm{NS}}, \quad \delta_{\Lambda} \mathbf{C}=\left(d \boldsymbol{\Lambda}-G^{(0)} \Lambda^{\mathrm{NS}}\right) \wedge \mathbf{e}^{B}, \quad \boldsymbol{\Lambda}=\sum_{n=0,1 / 2}^{4,7 / 2} \Lambda^{(2 n)}
$$

Наконец, имеется целый ряд $\mathbb{Z}_{2}$-симметрий, но они справедливы в теории IIA только в случае, когда $G^{(0)}=m=0$ (эти симметрии на мировом листе струны выписаны в работе [1]). Ниже показано, как эти симметрии действия преобразуют поля супергравитации. Если оба сектора IIA и IIB безмассовы, то существует симметрия относительно 
фермионного числа $(-1)^{F_{L}}$, задаваемая преобразованиями

$$
\begin{gathered}
\left\{\phi, g_{\mu \nu}, B_{\mu \nu}\right\} \rightarrow\left\{\phi, g_{\mu \nu}, B_{\mu \nu}\right\}, \\
\left\{C_{\mu_{1} \ldots \mu_{2 n-1}}^{(2 n-1)}\right\} \rightarrow-\left\{C_{\mu_{1} \ldots \mu_{2 n-1}}^{(2 n-1)}\right\}, \\
\left\{\psi_{\mu}, \lambda, \epsilon\right\} \rightarrow \begin{cases}+\mathcal{P}\left\{\psi_{\mu},-\lambda, \epsilon\right\} & \text { для IIA }, \\
+\mathcal{P}\left\{\psi_{\mu}, \lambda, \epsilon\right\} & \text { для IIB. }\end{cases}
\end{gathered}
$$

В случае теории IIB имеет место дополнительное преобразование четности $\Omega$ на мировом листе:

$$
\begin{gathered}
\left\{\phi, g_{\mu \nu}, B_{\mu \nu}\right\} \rightarrow\left\{\phi, g_{\mu \nu},-B_{\mu \nu}\right\}, \\
\left\{C_{\mu_{1} \ldots \mu_{2 n-1}}^{(2 n-1)}\right\} \rightarrow(-1)^{n+\frac{1}{2}}\left\{C_{\mu_{1} \ldots \mu_{2 n-1}}^{(2 n-1)}\right\}, \\
\left\{\psi_{\mu}, \lambda, \epsilon\right\} \rightarrow \sigma^{1}\left\{\psi_{\mu}, \lambda, \epsilon\right\} .
\end{gathered}
$$

В случае безмассовой теории типа IIA сушествует схожая $I_{9} \Omega$-симметрия, включаюшая дополнительное преобразование четности в направлении 9-й координаты. Выделяя эту координату из полного набора $\mu=(\underline{\mu}, \dot{9})$, получим соответствуюшее преобразование:

$$
\begin{gathered}
x^{\dot{9}} \rightarrow-x^{\dot{9},} \\
\left\{\phi, g_{\underline{\mu_{\nu}}}, B_{\underline{\mu \nu}}\right\} \rightarrow\left\{\phi, g_{\underline{\mu_{\nu}}},-B_{\underline{\mu \nu}}\right\}, \\
\left\{C_{\underline{\mu_{1} \cdots \underline{\mu_{2 n-1}}}}^{(2 n-1)}\right\} \rightarrow(-1)^{n+1}\left\{C_{\underline{\mu_{1} \cdots \underline{\mu_{2 n-1}}}}^{(2 n-1)}\right\}, \\
\left\{\psi_{\underline{\mu}}, \lambda, \epsilon\right\} \rightarrow+\Gamma^{9}\left\{\psi_{\underline{\mu}},-\lambda, \epsilon\right\} .
\end{gathered}
$$

Четность полей с одним и более индексами в направлении $\dot{9}$-й координаты задается следуюшим правилом: каждый индекс $\dot{9}$-й координаты в дополнение к указанным выше правилам порождает дополнительный знак минус.

Обе теории IIA и IIB, очевидно, симметричны относительно замены знаков всех фермионных полей на обратные (бозонные поля не преобразуются).

$\mathbb{Z}_{2}$-симметрии используются при построении суперструнных теорий с шестнадшатью суперзарядами (см. [25]). Симметрия $(-1)^{F_{L}}$ обеспечивает проекцию на теорию $E_{8} \times E_{8}$ гетеротической струны (типа IIA) или на теорию $S O(32)$ гетеротической струны (типа IIB). Симметрия $\Omega$ редуцирует теорию типа IIB на $S O(32)$ суперструну типа I, в то время как симметрия $I_{9} \Omega$ редуцирует теорию типа IIA к теории $S O(16) \times S O(16)$ суперструны типа I'.

По сравнению с обычной формулировкой супергравитации обобшенное псевдодействие (2.3) обладает тем преимушеством, что ценой наложения внешнего условия дуальности можно реализовать равноправие R-R-полей в действии. Мы приводим только кинетические члены действия, расширенньй набор полей не позволяет включить в действие член Черна-Саймонса. Как легко видеть, все кинетические члены переходят друг 
в друга при преобразованиях $T$-дуальности [26]. То же верно и для условий дуальности. Несмотря на определенную элегантность построенного действия, в его рамках невозможно построить настоящее действие из-за удвоения числа степеней свободы. Тем самым получаем, что такая демократическая формулировка непригодна для описания бранных действий, присоединенных к системе в объеме, по двум причинам. Во-первых, полная система инвариантна относительно преобразования $I_{9} \Omega$-симметрии только при $G^{(0)}=0$, но эта симметрия необходима для нашего построения системы "объем-

8-брана". Во-вторых, для описания заряженной доменной стенки необходимо, чтобы переменная $G^{(0)}$ имела противоположные значения с двух сторон от доменной стенки, т.е. необходимо, чтобы массовый параметр был бы лишь кусочно-постоянен. Поэтому R-R-равноправие должно быть нарушено. Мы рассматриваем это в следующих двух пунктах.

2.2. Дуальная формулировка теории IIA. В этом пункте приводится новая дуальная формулировка с действием, пригодным лишь для теории IIA. В рамках этого подхода удается построить настоящее действие, и оно используется при построении связанной системы "объем-брана". Мы называем этот подход дуальной формулировкой, смысл этого термина разъясняется в следуюшем пункте.

В новой формулировке независимыми являются поля

$$
\left\{e_{\mu}^{a}, B_{\mu \nu}, \phi, G^{(0)}, G_{\mu \nu}^{(2)}, G_{\mu_{1} \ldots \mu_{4}}^{(4)}, A_{\mu_{1} \ldots \mu_{5}}^{(5)}, A_{\mu_{1} \ldots \mu_{7}}^{(7)}, A_{\mu_{1} \ldots \mu_{9}}^{(9)}, \psi_{\mu}, \lambda\right\} .
$$

Действие в объеме имеет вид

$$
\begin{aligned}
S_{\text {bulk }}= & -\frac{1}{2 \kappa_{10}^{2}} \int d^{10} x \sqrt{-g}\left\{e ^ { - 2 \phi } \left[R(\omega(e))-4(\partial \phi)^{2}+\frac{1}{2} H \cdot H-2 \partial^{\mu} \phi \chi_{\mu}^{(1)}+\right.\right. \\
& \left.+H \cdot \chi^{(3)}+2 \bar{\psi}_{\mu} \Gamma^{\mu \nu \rho} \nabla_{\nu} \psi_{\rho}-2 \bar{\lambda} \Gamma^{\mu} \nabla_{\mu} \lambda+4 \bar{\lambda} \Gamma^{\mu \nu} \nabla_{\mu} \psi_{\nu}\right]+ \\
& +\sum_{n=0,1,2}\left(\frac{1}{2} G^{(2 n)} \cdot G^{(2 n)}+G^{(2 n)} \cdot \Psi^{(2 n)}\right)- \\
& -\star\left[\frac{1}{2} G^{(4)} G^{(4)} B-\frac{1}{2} G^{(2)} G^{(4)} B^{2}+\frac{1}{6} G^{(2) 2} B^{3}+\frac{1}{6} G^{(0)} G^{(4)} B^{3}-\right. \\
& \left.\left.-\frac{1}{8} G^{(0)} G^{(2)} B^{4}+\frac{1}{40} G^{(0) 2} B^{5}+\mathbf{e}^{-B} \mathbf{G} d\left(A^{(5)}-A^{(7)}+A^{(9)}\right)\right]\right\}+ \\
& + \text { члены четвертого порядка по фермионам, }
\end{aligned}
$$

где все знаки $\wedge$ в топологическом члене (отмеченном звездочкой) опушены. В последнем члене также подразумевается проекция на 10 -форму. Форма $\mathbf{G}$ определена так же, как в (2.4), но теперь $G^{(0)}, G^{(2)}$ и $G^{(4)}$ суть независимые поля (назьваемые чернылми ящиками); никаких условий вида (2.5) не предполагается. Тождества Бианки для этих полей вводятся с помошью множителей Лагранжа $A^{(9)}, A^{(7)}$ и $A^{(5)}$. Напряженность поля NS-NS 3-формы задается формулой (2.5). 
Симметрии действия сходны с симметриями в демократической формулировке с некоторыми изменениями. В преобразованиях суперсимметрии для гравитино и гаугино суммирование теперь идет только по $n=0,1,2$ :

$$
\begin{aligned}
\delta_{\epsilon} e_{\mu}{ }^{a} & =\bar{\epsilon} \Gamma^{a} \psi_{\mu} \\
\delta_{\epsilon} \psi_{\mu} & =\left(\partial_{\mu}+\frac{1}{4} \psi_{\mu}+\frac{1}{8} \Gamma_{11} \not H_{\mu}\right) \epsilon+\frac{1}{8} e^{\phi} \sum_{n=0,1,2} \frac{1}{(2 n) !} G^{(2 n)} \Gamma_{\mu}\left(\Gamma_{11}\right)^{n} \epsilon, \\
\delta_{\epsilon} B_{\mu \nu} & =-2 \bar{\epsilon} \Gamma_{[\mu} \Gamma_{11} \psi_{\nu]}, \\
\delta_{\epsilon} \lambda & =\left(\not \partial \phi-\frac{1}{12} \Gamma_{11} \not H\right) \epsilon+\frac{1}{4} e^{\phi} \sum_{n=0,1,2} \frac{5-2 n}{(2 n) !} G^{(2 n)}\left(\Gamma_{11}\right)^{n} \epsilon, \\
\delta_{\epsilon} \phi & =\frac{1}{2} \bar{\epsilon} \lambda, \\
\delta_{\epsilon} \mathbf{A} & =\mathbf{e}^{-B} \wedge \mathbf{E}, \\
\delta_{\epsilon} \mathbf{G} & =d \mathbf{E}+\mathbf{G} \wedge \delta_{\epsilon} B-H \wedge \mathbf{E},
\end{aligned}
$$

где

$$
E_{\mu_{1} \ldots \mu_{2 n-1}}^{(2 n-1)} \equiv-e^{-\phi} \bar{\epsilon} \Gamma_{\left[\mu_{1} \ldots \mu_{2 n-2}\right.}\left(\Gamma_{11}\right)^{n}\left((2 n-1) \psi_{\left.\mu_{2 n-1}\right]}-\frac{1}{2} \Gamma_{\left.\mu_{2 n-1}\right]} \lambda\right) .
$$

Преобразования черных ящиков $\mathbf{G}$ следуют из условия, что комбинация $\mathbf{e}^{-B} \mathbf{G}$ преобразуется в полную производную. Используются формальные суммы

$$
\mathbf{A}=\sum_{n=1}^{5} A^{(2 n-1)}, \quad \mathbf{E}=\sum_{n=1}^{5} E^{(2 n-1)}, \quad \mathbf{G}=\sum_{n=0}^{5} G^{(2 n)}
$$

Заметим, что первая формальная сумма в (2.13) содержит поля $\left(A^{(1)}\right.$ и $\left.A^{(3)}\right)$, которые не входят в действие. То же самое верно и для формы $\mathbf{G}$, которая содержит дополнительные поля $G^{(6)}, G^{(8)}$ и $G^{(10)}$. Хотя эти поля и не входят в действие, с использованием правил суперсимметрии (2.12) и уравнений движения, следующих из действия (2.11), можно показать, что эти поля участвуют в алгебре суперсимметрии.

Калибровочные преобразования с параметрами $\Lambda^{\mathrm{NS}}$ и $\Lambda^{(2 n)}$ имеют вид

$$
\begin{aligned}
& \delta_{\Lambda} B=d \Lambda^{\mathrm{NS}}, \quad \delta_{\Lambda} \mathbf{A}=d \mathbf{\Lambda}-G^{(0)} \Lambda^{\mathrm{NS}}-d \Lambda^{\mathrm{NS}} \wedge \mathbf{A}, \\
& \delta_{\Lambda} \mathbf{G}=d \Lambda \wedge\left(\mathbf{G}-\mathbf{e}^{B} \wedge\left(d \mathbf{A}+G^{(0)}\right)\right)+\mathbf{e}^{B} \wedge \Lambda \wedge d G^{(0)} .
\end{aligned}
$$

Отметим, что по отношению к R-R-преобразованиям потенциалы $\mathbf{A}$ преобразуются как полные производные, а черные ящики оказываются инвариантными.

Наконец, сушествуют еше и $\mathbb{Z}_{2}$-симметрии $(-1)^{F_{L}}$ и $I_{9} \Omega$, относительно которых действие инвариантно. В отличие от демократической формулировки, эти две $\mathbb{Z}_{2}$-симметрии остаются симметриями и при $G^{(0)} \neq 0$. Симметрия $(-1)^{F_{L}}$ задается отображениями

$$
\begin{gathered}
\left\{\phi, g_{\mu \nu}, B_{\mu \nu}\right\} \rightarrow\left\{\phi, g_{\mu \nu}, B_{\mu \nu}\right\}, \\
\left\{G_{\mu_{1} \ldots \mu_{2 n}}^{(2 n)}, A_{\mu_{1} \ldots \mu_{2 n-1}}^{(2 n-1)}\right\} \rightarrow-\left\{G_{\mu_{1} \ldots \mu_{2 n}}^{(2 n)}, A_{\mu_{1} \ldots \mu_{2 n-1}}^{(2 n-1)}\right\}, \\
\left\{\psi_{\mu}, \lambda, \epsilon\right\} \rightarrow+\Gamma_{11}\left\{\psi_{\mu},-\lambda, \epsilon\right\},
\end{gathered}
$$


а $I_{9} \Omega$-симметрия есть

$$
\begin{gathered}
x^{9} \rightarrow-x^{9}, \\
\left\{\phi, g_{\underline{\mu \nu}}, B_{\underline{\mu \nu}}\right\} \rightarrow\left\{\phi, g_{\underline{\mu \nu}},-B_{\underline{\mu \nu}}\right\}, \\
\left\{G_{\underline{\mu_{1} \cdots \underline{\mu_{2 n}}}}^{(2 n)}, A_{\underline{\mu_{1} \cdots \mu_{2 n-1}}}^{(2 n-1)}\right\} \rightarrow(-1)^{n+1}\left\{G_{\underline{\mu_{1} \cdots \underline{\mu_{2 n}}}}^{(2 n)}, A_{\underline{\mu_{1} \cdots \underline{\mu_{2 n-1}}}}^{(2 n-1)}\right\}, \\
\left\{\psi_{\underline{\mu}}, \lambda, \epsilon\right\} \rightarrow+\Gamma^{9}\left\{\psi_{\underline{\mu}},-\lambda, \epsilon\right\} .
\end{gathered}
$$

2.3. Стандартная формулировка теории IIA. Построенное дуальное действие (2.11) может быть преобразовано к струнной версии стандартного действия массивной IIA супергравитации (первоначально найденной в системе Эйнштейна в работе [4]). Покажем, как преобразовать одну формулировку в другую.

Рассмотрим полевые уравнения для потенциалов $A^{(5)}, A^{(7)}$ и $A^{(9)}$, задаваемых уравнением

$$
d\left(\mathbf{e}^{-B} \wedge \mathbf{G}\right)=0
$$

Наиболее общие решения записываются в виде

$$
\mathbf{e}^{-B} \wedge \mathbf{G}=d \mathbf{A}+\mathbf{G}_{\text {flux }}
$$

или более явно

$$
\begin{aligned}
& G^{(0)}=G_{\text {flux }}^{(0)}, \quad G^{(2)}=d A^{(1)}+G^{(0)} B+G_{\text {flux }}^{(2)}, \\
& G^{(4)}=d A^{(3)}+G^{(2)} \wedge B-\frac{1}{2} G^{(0)} B \wedge B+G_{\text {flux }}^{(4)},
\end{aligned}
$$

где $\mathbf{G}_{\text {flux }}$ - когомологические решения. Если имеет место полная десятимерная лоренцева симметрия, то только лишь поток $G_{\text {flux }}^{(0)}$ может быть отличен от нуля; этот поток должен быть постоянным и совпадать с массовым параметром $m$ теории Романса. Мы рассматриваем в основном именно такую ситуацию. Отметим тем не менее, что можно было бы считать, что в нашей конфигурации помимо $m$ присутствуют и постоянные потоки $G_{\text {flux }}^{(2)}$ и $G_{\text {flux }}^{(4)}$ и что при этом сохраняется только четырехмерная лоренцева симметрия (см., например, [27], [28]).

Здесь и ниже положим

$$
G_{\text {flux }}^{(0)}=m, \quad G_{\text {flux }}^{(2)}=G_{\text {flux }}^{(4)}=0 .
$$

Подставляя эти решения в объемное действие (2.11), получим теорию без черных ящиков, но с массовым параметром, 1-формой и 3-формой. Отметим, что полевой состав этой теории (2.1) совпадает с полевым составом теории Романса. Анализ действия показывает, что оно совпадает со стандартным действием теории Романса, записанным в А-базисе (2.6).

Заметим, что, как было показано, базис (2.6) есть в точности комбинация полей, возникаюшая в члене Весса-Зумино D-бранного действия, в котором поля мирового объема струны положены равными нулю. 
В C-базисе действие в стандартной формулировке имеет вид

$$
\begin{aligned}
S_{\text {bulk }}= & -\frac{1}{2 \kappa_{10}^{2}} \int d^{10} x \sqrt{-g}\left\{e ^ { - 2 \phi } \left[R(\omega(e))-4(\partial \phi)^{2}+\frac{1}{2} H \cdot H-2 \partial^{\mu} \phi \chi_{\mu}^{(1)}+\right.\right. \\
& \left.+H \cdot \chi^{(3)}+2 \bar{\psi}_{\mu} \Gamma^{\mu \nu \rho} \nabla_{\nu} \psi_{\rho}-2 \bar{\lambda} \Gamma^{\mu} \nabla_{\mu} \lambda+4 \bar{\lambda} \Gamma^{\mu \nu} \nabla_{\mu} \psi_{\nu}\right]+ \\
& +\sum_{n=0,1,2}\left(\frac{1}{2} G^{(2 n)} \cdot G^{(2 n)}+G^{(2 n)} \cdot \Psi^{(2 n)}\right)- \\
& \left.-\star\left[\frac{1}{2} d C^{(3)} d C^{(3)} B+\frac{1}{6} G^{(0)} d C^{(3)} B^{3}+\frac{1}{40} G^{(0) 2} B^{5}\right]\right\}+ \\
& + \text { члены четвертого порядка по фермионам, }
\end{aligned}
$$

где в топологическом члене (отмеченном звездочкой) опущены все символы $\wedge$. Здесь все напряженности полей $G^{(2 n)}$ заданы формулами $(2.5)$, а $G^{(0)}$ - постоянное поле. Шесть членов Черна-Саймонса в формуле (2.11) могут быть вьписаны в виде трех членов в случае, если $G^{(2 n)}$ суть напряженности полей. Стандартное действие типа IIA инвариантно относительно преобразований $N=2$ суперсимметрии

$$
\begin{aligned}
\delta_{\epsilon} e_{\mu}{ }^{a}= & \bar{\epsilon} \Gamma^{a} \psi_{\mu}, \\
\delta_{\epsilon} \psi_{\mu}= & \left(\partial_{\mu}+\frac{1}{4} \psi_{\mu}+\frac{1}{8} \Gamma_{11} \not H_{\mu}\right) \epsilon+\frac{1}{8} e^{\phi} \sum_{n=0,1,2} \frac{1}{(2 n) !} G^{(2 n)} \Gamma_{\mu}\left(\Gamma_{11}\right)^{n} \epsilon, \\
\delta_{\epsilon} B_{\mu \nu}= & -2 \bar{\epsilon} \Gamma_{[\mu} \Gamma_{11} \psi_{\nu]}, \\
\delta_{\epsilon} C_{\mu_{1} \ldots \mu_{2 n-1}}^{(2 n-1)}= & -e^{-\phi} \bar{\epsilon} \Gamma_{\left[\mu_{1} \ldots \mu_{2 n-2}\right.}\left(\Gamma_{11}\right)^{n}\left((2 n-1) \psi_{\left.\mu_{2 n-1}\right]}-\frac{1}{2} \Gamma_{\left.\mu_{2 n-1}\right]} \lambda\right)+ \\
& +(n-1)(2 n-1) C_{\left[\mu_{1} \ldots \mu_{2 n-3}\right.}^{(2 n-3)} \delta_{\epsilon} B_{\left.\mu_{2 n-2} \mu_{2 n-1}\right]}, \\
\delta_{\epsilon} \lambda= & \left(\not \partial \phi+\frac{1}{12} \not H \Gamma_{11}\right) \epsilon+\frac{1}{4} e^{\phi} \sum_{n=0,1,2} \frac{5-2 n}{(2 n) !} G^{(2 n)}\left(\Gamma_{11}\right)^{n} \epsilon, \\
\delta_{\epsilon} \phi= & \frac{1}{2} \bar{\epsilon} \lambda
\end{aligned}
$$

и относительно калибровочных преобразований (2.8). Заметим, что $\mathbb{Z}_{2}$-симметрии (2.9) и (2.10) сохраняются только при нулевом массовом параметре.

Таким образом, дуальная формулировка теории из п. 2.2 может быть преобразована к стандартной формулировке. Также можно использовать теорию Романса для вывода действия (2.11). Первый шаг в этом направлении состоит в “дуализации” 1- и 3-форм. Тогда полевые напряженности преврашаются в черные ящики, а тождества Бианки накладываются с помошью множителей Лагранжа; при этом поле $G^{(0)}$ интерпретируется как напряженность поля 0-формы. Полная дуализация достигается, если мы устраним все черные яшики, разрешая уравнения движения. Уже после этого первого шага получается действие (2.11), которое назьвается дуальной формулировкой. 
Тем самым мы имеем три различных формулировки одной и той же теории. Демократическая формулировка включает в себя все потенциалы, но поскольку в этой формулировке отсутствует настоящее действие, она не может быть использована для добавления бранных действий. Стандартная формулировка приводит к настояшему действию, содержашему напряженности $C^{(1)}$ и $C^{(3)}$, а потому пригодна для описания 0- и 2-бран. Дуальное действие содержит дуальные потенциалы $A^{(5)}, A^{(7)}$ и $A^{(9)}$, соответствующие 4-, 6- и 8-бранам. Используя две последние формулировки, можно присоединять бранные действия любой (четной) размерности до тех пор, пока одновременно не присоединятся браны низших и высших размерностей.

\section{3. СУПЕРСИММЕТРИЯ НА БРАНЕ}

Таким образом, мы построили суперсимметрию в объеме, а теперь обратимся к суперсимметрии на бране. Как отмечалось во введении, наибольший интерес представляют собой одномерные орбифолдные конструкции с 8-бранами в орбифолдных точках. Используя технику 3-браны на орбифолде в размерности $D=5$ [11], мы рассчитьваем построить ориентифолд с помощью $\mathbb{Z}_{2}$-симметрии объемного действия. Бранные действия присоединяются в фиксированных точках, и эти действия оказываются инвариантными относительно действия редуцированной $(N=1)$ суперсимметрии. В этом случае можно снять ограничение теории на доменные стенки (в данном случае 8-браны), поскольку методы бранного анализа совпадают для всех ориентифолдов низших размерностей.

\section{$\mathbb{Z}_{2}$-симметрии, используемые в ориентифолдной конструкции на $\mathrm{O} p$-плоскости. Каждое преобразование $T$-дуальности между струнами IIA и IIB в низших размерностях индуцирует преобразование $(-1)^{F_{L}}$.}

\begin{tabular}{|c|c|c|}
\hline$p$ & IIB & IIA \\
\hline 9 & $\Omega$ & - \\
8 & - & $I_{9} \Omega$ \\
7 & $(-1)^{F_{L}} I_{9,8} \Omega$ & - \\
6 & - & $(-1)^{F_{L}} I_{9,8,7} \Omega$ \\
5 & $I_{9,8, \ldots, 6} \Omega$ & - \\
4 & - & $I_{9,8, \ldots, 5} \Omega$ \\
3 & $(-1)^{F_{L}} I_{9,8, \ldots, 4} \Omega$ & - \\
2 & - & $(-1)^{F_{L}} I_{9,8, \ldots, 3} \Omega$ \\
1 & $I_{9,8, \ldots, 2} \Omega$ & - \\
0 & - & $I_{9,8, \ldots, 1} \Omega$ \\
\hline
\end{tabular}


В предыдущем разделе было показано, что объемное действие обладает целым набором симметрий, включая пространственную четность. Для того чтобы построить ориентифолд, подходящая $\mathbb{Z}_{2}$-симметрия должна содержать операцию четности относительно поперечного направления. Более того, для построения заряженной доменной стенки необходимо, чтобы R-R-потенциал $(p+1)$-формы был четным относительно $p$-браны. Для 8-браны указанными свойствами обладает $I_{9} \Omega$-симметрия. Для других $p$-бран представляется естественным использовать $\mathbb{Z}_{2}$-симметрию

$$
I_{9,8, \ldots, p+1} \Omega \equiv\left(I_{9} \Omega\right)\left(I_{8} \Omega\right) \ldots\left(I_{p+1} \Omega\right)
$$

в которой $I_{q} \Omega$ есть преобразование (2.10) с заменой индекса 9 на $q$, и преобразования $I_{q}$ и $\Omega$ коммутируют. Однако для некоторых $p$-бран $(p=2,3,6,7)$ соответствуюшие $C^{(p+1)}$ $\mathrm{R}$-R-потенциалы нечетны относительно таких $\mathbb{Z}_{2}$-симметрий. При этом, чтобы получить правильную четность, необходимо ввести дополнительное преобразование $(-1)^{F_{L}}$, которое также следует из $T$-дуальности [29]. Это приводит к $\mathbb{Z}_{2}$-симметрии для каждой $p$-браны, приведенной в таблице.

Правильное преобразование $\mathbb{Z}_{2}$-симметрии для IIA $\mathrm{O} p$-плоскости обшего положения имеет вид

$$
\left((-1)^{F_{L}}\right)^{\frac{p}{2}} I_{9,8, \ldots, p+1} \Omega .
$$

Действие этой $\mathbb{Z}_{2}$-симметрии на поля в объеме имеет вид (подчеркнутые индексы относятся к направлениям мирового объема, т.е. $\mu=(\underline{\mu}, p+1, \ldots, 9))$

$$
\begin{aligned}
& \left\{x^{p+1}, \ldots, x^{9}\right\} \rightarrow-\left\{x^{p+1}, \ldots, x^{9}\right\}, \\
& \left\{\phi, g_{\underline{\mu \nu}}, B_{\underline{\mu \nu}}\right\} \rightarrow\left\{\phi, g_{\underline{\mu \nu}},-B_{\underline{\mu \nu}}\right\}, \\
& \left\{A_{\underline{\mu_{1}} \ldots \underline{\mu_{5}}}^{(5)}, A_{\underline{\mu_{1}} \ldots \underline{\mu_{9}}}^{(9)}, G_{\underline{\mu \nu}}^{(2)}\right\} \rightarrow(-1)^{\frac{p}{2}}\left\{A_{\underline{\mu_{1}} \ldots \underline{\mu_{5}}}^{(5)}, A_{\underline{\mu_{1}} \ldots \underline{\mu_{9}}}^{(9)}, G_{\underline{\mu \nu}}^{(2)}\right\}, \\
& \left\{A_{\underline{\mu_{1} \ldots} \underline{\mu_{7}}}^{(7)}, G^{(0)}, G_{\underline{\mu_{1}} \ldots \underline{\mu_{4}}}^{(4)}\right\} \rightarrow(-1)^{\frac{p}{2}+1}\left\{A_{\underline{\mu_{1} \ldots} \ldots \underline{\mu_{7}}}^{(7)}, G^{(0)}, G_{\underline{\mu_{1} \ldots \underline{\mu_{4}}}}^{(4)}\right\}, \\
& \left\{\psi_{\underline{\mu}}, \epsilon\right\} \rightarrow-\alpha \Gamma^{(p+1) \ldots 9}\left(-\Gamma_{11}\right)^{\frac{p}{2}}\left\{\psi_{\underline{\mu}}, \epsilon\right\}, \\
& \{\lambda\} \rightarrow+\alpha \Gamma^{(p+1) \ldots 9}\left(+\Gamma_{11}\right)^{\frac{p}{2}}\{\lambda\},
\end{aligned}
$$

а для полей с другими индексами надо добавить дополнительный знак минус при каждой замене индекса мирового объема $\underline{\mu}$ на индекс в перпендикулярном направлении. Остается возможность комбинирования этой симметрии с изменением знака всех фермионов. Эта симметрия вводит число $\alpha= \pm 1$ в указанные выше правила. Симметрия (3.1) используется при построении ориентифолдов.

Для такого построения выберем пространство-время в виде $\mathcal{M}^{p+1} \times T^{9-p}$. Радиусы тора, которые могут зависеть от координат пространственного объема, обозначим $R^{\bar{\mu}}$. Все поля удовлетворяют условиям периодичности

$$
\Phi\left(x^{\bar{\mu}}\right)=\Phi\left(x^{\bar{\mu}}+2 \pi R^{\bar{\mu}}\right),
$$


где $\bar{\mu}=(p+1, \ldots, 9)$. Здесь присутствуют только те поля, которые четны относительно соответствуюшего преобразования $\mathbb{Z}_{2}$-симметрии (3.1). Это устанавливает соответствие между полями в точках $x^{\bar{\mu}}$ и $-x^{\bar{\mu}}$ в объеме. В особой (фиксированной) точке ориентифолда это соотношение становится локальным и выделяет половину полей. Тем самым в фиксированных точках вставок бран остается только $N=1$ суперсимметрия. Рассмотрим пример девятимерного ориентифолда. Проекция редуцирует объемную $N=2$ суперсимметрию до $N=1$ суперсимметрии на бране. При этом лиш половина из 32 компонент поля $\epsilon$ оказываются четными относительно преобразований (3.2).

Первоначальный состав полей - $(128+128)$ мультиплет $D=10, N=2$ супергравитации - редуцируется на бране до приводимого $(64+64)$ мультиплета $D=9, N=1$ теории, состоящей из супергравитации и присоединенного векторного мультиплета. Можно проводить редукцию и дальше до постоянного тора. Этот частный выбор пространства-времени затем выделяет $N=1(8+8)$ векторный мультиплет (содержащий $\left.e_{\dot{9}}{ }^{9}\right)$ и инвариантный неприводимый $(56+56)$ мультиплет $D=9, \quad N=1$ супергравитации. Сходные редукции происходят в ориентифолдах низших размерностей, в которых $(64+64) N=1$ теория также состоит из набора мультиплетов.

Предполагается, что $p$-бранное действие $(p=0,2,4,6,8)$ пропорционально лагранжиану

$\mathcal{L}_{p}=-e^{-\phi} \sqrt{-g_{(p+1)}}-\alpha \frac{1}{(p+1) !} \varepsilon^{(p+1)} C^{(p+1)}, \quad \varepsilon^{(p+1)} C^{(p+1)} \equiv \varepsilon_{\underline{\mu_{0}} \cdots \underline{\mu_{p}}}^{(p+1)} C^{(p+1) \underline{\mu_{0}} \cdots \underline{\mu_{p}}}$, где $\varepsilon^{(p+1)} \underline{\mu_{0}} \cdots \underline{\mu_{p}}=\varepsilon^{(10)} \underline{\mu_{0}} \cdots \underline{\mu_{p}}(p \dot{+1}) \ldots \dot{9}$, что следует из условия $e_{\underline{\mu}}{ }^{\bar{a}}=0$ (ввиду нечетности). Подчеркнутые индексы принимают $p+1$ значение и относятся к пространственному объему. Параметр $\alpha$ такой же, как в преобразованиях (3.2), и равен +1 для бран, знаки натяжения и заряда которых по определению совпадают, и -1 для антибран, у которых знаки натяжения и заряда противоположны. Заметим, что поскольку $B$ обращается в нуль на бране, потенциалы $C^{(p+1)}$ и $A^{(p+1)}$ равны. Легко показать, что действие $p$-браны инвариантно относительно подходяшей $N=1$ суперсимметрии:

$$
\delta_{\epsilon} \mathcal{L}_{p}=-e^{-\phi} \sqrt{-g_{(p+1)}} \bar{\epsilon}\left(1-\alpha \Gamma^{(p+1) \ldots 9}\left(\Gamma_{11}\right)^{\frac{p}{2}}\right) \Gamma \underline{\mu}\left(\psi_{\underline{\mu}}-\frac{1}{18} \Gamma_{\underline{\mu}} \lambda\right) .
$$

Вариация действия обращается в нуль, поскольку в преобразованиях (3.2) присутствует проекция, которая выделяет браны или антибраны в зависимости от знака $\alpha$ (соответственно +1 или -1$)$. В последующем обсуждении предполагается, что $\alpha=1$, но противоположный случай полностью аналогичен, надо лишь заменить браны на антибраны.

В теории с обрезанием можно построить бранное действие, которое состоит только из бозонов и все еше оказывается суперсимметричным. После этого можно ввести члены источников для различных потенциалов. В общем случае имеются $2^{9-p}$ фиксированных точек. Компактность поперечного пространства приводит к тому, что полный заряд равен нулю. Тогда полное действие имеет вид

$$
\begin{gathered}
\mathcal{L}=\mathcal{L}_{\mathrm{bulk}}+k_{p} \mathcal{L}_{p} \Delta_{p} \\
\Delta_{p} \equiv\left(\delta\left(x^{p+1}\right)-\delta\left(x^{p+1}-\pi R^{p+1}\right)\right) \ldots\left(\delta\left(x^{9}\right)-\delta\left(x^{9}-\pi R^{9}\right)\right),
\end{gathered}
$$


в котором браны в фиксированных точках имеют натяжение и заряд, пропорциональные $\pm k_{p}$ (параметр размерности $1 /[\text { длина }]^{p+1}$ ). Поскольку антибраны не удовлетворяют условиям суперсимметрии (3.3), необходимо включать браны с положительным и отрицательным натяжениями, чтобы получить нулевой полный заряд. Мы интерпретируем браны с отрицательным натяжением как О-плоскости.

Уравнения движения, следующие из действия (3.4), приводят к появлению $\delta$-функции в тождествах Бианки для напряженности поля $(8-p)$-формы. В общем случае красивые решения найти довольно трудно, но ситуация упрошается в частном случае 8-браны, исследуемом в работе [1] на основе вышеприведенной новой формулировки $D=10$ суперсимметрии.

\section{4. СВОДКА РЕЗУЛЬТАТОВ И ОБСУЖДЕНИЕ}

Основным результатом настояшей работы является новая формулировка $D=10$ супергравитации типа II (раздел 2). Как для теорий типа IIA, так и для IIB теорий построены демократические теории в объеме, в которых различные R-R-потенциалы рассматриваются равноправными. Поскольку при этом число степеней свободы в R-R-секторе удваивается, необходимо наложить дополнительные соотношения дуальности, и построение настояшего действия при этом становится невозможным. Мы обсуждаем возможность построения так называемого псевдодействия, содержащего кинетический член для всех потенциалов, но без члена Черна-Саймонса. Более того, мы явно нарушили самодуальность в случае теории IIA, что открыло путь к построению настоящего действия. Отнюдь не все R-R-потенциалы, а только половина напряженностей $C^{(p)}$ появляются в действии. Обсуждались как стандартная $(p=1,3)$, так и дуальная $(p=5,7,9)$ формулировки. С помощью этих действий можно описать все системы типа "объем-

брана".

В разделе 3 исследовались бранные действия, присоединенные в фиксированных точках ориентифолдов. Все бранные действия сохраняют на соответствующем ориентифолде половину $N=2$ суперсимметрии. Этому условию удовлетворяют либо браны, либо антибраны, но не те и другие вместе. Это также можно понять исходя из суперсимметричного баланса сил. Чтобы получить исчезающий заряд в компактном пространстве, надо использовать браны (антибраны) как с положительным, так и с отрицательным натяжениями. Последние могут быть интерпретированы как ориентифолдные плоскости, в то время как первые отвечают бранам Дирихле. Частный случай таких бран (8-брана) был детально исследован в работе [1]. Наше суперсимметричное действие объем-брана дает описание системы D8-O8. Это фундаментальные объекты в струнной теории типа I'.

Благодарности. Работа поддержана NSF (грант PHY-9870115).

\section{Список литературы}

[1] E. Bergshoeff, R. Kallosh, T. Ortín, D. Roest, and A. Van Proeyen. New formulations of $D=10$ supersymmetry and D8-O8 domain walls. hep-th/0103233.

[2] J. Polchinski. String Theory. Cambridge: Cambridge Univ. Press, 1999. 
[3] J. Polchinski. Phys. Rev. Lett. 1995. V. 75. P. 4724; hep-th/9510017.

[4] L. J. Romans. Phys. Lett. B. 1986. V. 169. P. 374.

[5] J. Polchinski and E. Witten. Nucl. Phys. B. 1996. V. 460. P. 525; hep-th/9510169.

[6] E. Bergshoeff, M. de Roo, M. B. Green, G. Papadopoulos, and P. K. Townsend. Nucl. Phys. B. 1996. V. 470. P. 113; hep-th/9601150.

[7] M. J. Duff and P. van Nieuwenhuizen. Phys. Lett. B. 1980. V. 94. P. 179.

[8] A. Aurilia, H. Nicolai, and P. K. Townsend. Nucl. Phys. B. 1980. V. 176. P. 509.

[9] N. Alonso-Alberca, P. Meessen, and T. Ortín. Phys. Lett. B. 2000. V. 482. P. 400; hep-th/0003248.

[10] L. Randall and R. Sundrum. Phys. Rev. Lett. 1999. V. 83. P. 3370; hep-ph/9905221; Phys. Rev. Lett. 1999. V. 83. P. 4690; hep-th/9906064.

[11] E. Bergshoeff, R. Kallosh, and A. Van Proeyen. JHEP. 2000. V. 10. P. 033; hep-th/0007044.

[12] E. Bergshoeff, H. J. Boonstra, and T. Ortín. Phys. Rev. D. 1996. V. 53. P. 7206; hep-th/9508091.

[13] M. B. Green, C. M. Hull, and P. K. Townsend. Phys. Lett. B. 1996. V. 382. P. 65; hep-th/9604119.

[14] M. Cederwall, A. von Gussich, B. E. W. Nilsson, P. Sundell, and A. Westerberg. Nucl. Phys. B. 1997. V. 490. P. 179; hep-th/9611159.

[15] E. Bergshoeff and P. K. Townsend. Nucl. Phys. B. 1997. V. 490. P. 145; hep-th/9611173.

[16] M. Perry and J. H. Schwarz. Nucl. Phys. B. 1997. V. 489. P. 47; hep-th/9611065.

[17] P. Pasti, D. Sorokin, and M. Tonin. Phys. Rev. D. 1995. V. 52. P. 4277; hep-th/9506109; Phys. Rev. D. 1997. V. 55. P. 6292; hep-th/9611100; Phys. Lett. B. 1997. V. 398. P. 41; hep-th/9701037.

[18] M. Huq and M. A. Namazie. Class. Q. Grav. 1985. V. 2. P. 293.

[19] F. Giani and M. Pernici. Phys. Rev. D. 1984. V. 30. P. 325.

[20] I. C. G. Campbell and P. C. West. Nucl. Phys. B. 1984. V. 243. P. 112.

[21] J. H. Schwarz. Nucl. Phys. B. 1983. V. 226. P. 269.

[22] P. S. Howe and P. C. West. Nucl. Phys. B. 1984. V. 238. P. 181.

[23] M. Fukuma, T. Oota, and H. Tanaka. Progr. Theor. Phys. 2000. V. 103. P. 425; hep-th/9907132.

[24] E. Bergshoeff, M. de Roo, B. Janssen, and T. Ortín. Nucl. Phys. B. 1999. V. 550. P. 289; hep-th/9901055.

[25] E. Bergshoeff, E. Eyras, R. Halbersma, J. P. van der Schaar, C. M. Hull, and Y. Lozano. Nucl. Phys. B. 2000. V. 564. P. 29; hep-th/9812224.

[26] P. Meessen and T. Ortín. Nucl. Phys. B. 1999. V. 541. P. 195; hep-th/9806120.

[27] J. Polchinski and A. Strominger. Phys. Lett. B. 1996. V. 388. P. 736; hep-th/9510227.

[28] S. Gukov. Nucl. Phys. B. 2000. V. 574. P. 169; hep-th/9911011.

[29] A. Dabholkar. Lectures on orientifolds and duality. In: High Energy Physics and Cosmology 1997. (Proc. Trieste 1997, ICTP Series in Theor. Phys. V. 14). Eds. E. Gava et al. Singapore: World Scientific, 1998. P. 128; hep-th/9804208. 